\title{
Chiral luminescent CdS nano-tetrapods $\dagger$
}

\author{
Joseph E. Govan, ${ }^{a b}$ Edward Jan, ${ }^{c}$ Ana Querejeta, ${ }^{c}$ Nicholas A. Kotov ${ }^{c}$ and \\ Yurii K. Gun'ko*ab
}

Received 14th April 2010, Accepted 16th June 2010

DOI: $10.1039 / \mathbf{c 0 c c 0 0 9 3 0 j}$

The utilisation of chiral penicillamine stabilisers allowed the preparation of new water soluble white emitting CdS nano-tetrapods, which demonstrated circular dichroism in the band-edge region of the spectrum.

Semiconductor nanocrystals or quantum dots are remarkable for their size dependent optical and photonic properties, which differ significantly from the bulk material. These unique properties are of great interest for a number of potential applications including biomedical imaging, ${ }^{1-4}$ sensing, ${ }^{5}$ light-emitting diodes (LEDs), ${ }^{6}$ photovoltaics ${ }^{7}$ and displays. ${ }^{8}$

Previously the preparation of new chiral quantum dots which demonstrate circular dichroism (CD) activity was achieved by the microwave synthesis in the presence of the chiral penicillamine stabiliser. ${ }^{9}$ In this case the formation of chiral defects in the nanocrystals structure leads both to the defect emission and CD response. ${ }^{10}$ More recent reports on chiral CdTe based nanoparticles have also been published. ${ }^{11,12}$ There is also a very recent theoretical work, which demonstrated that the electric field of the metal or semiconductor nanoparticle enhances the signal from the chiral stabiliser and other chiral species. ${ }^{13}$ Chirality is a common occurrence in the natural world and chiral compounds have very important applications in chemistry, biology, pharmacology and medicine. Therefore chiral nanoparticles have been envisaged to play an important role in science and technology. ${ }^{14,15}$ In particular CD active luminescent nanoparticles have a great potential for the development of new chiral nanosensors.

Over last years there have also been several reports on semiconductor nanomaterials with tetrapodal morphology. These amazing nanostructures have been of great interest due to their potential use in photovoltaics, ${ }^{16}$ in the synthesis of asymmetric nano-assemblies ${ }^{17}$ as well as prospective additives for polymer reinforcement. ${ }^{18}$ These materials have also displayed anisotropic emission ${ }^{19}$ as a direct result of their morphology. Normally the synthesis of nanotetrapods ${ }^{18,20-29}$ involves the use of high temperature organic phase reactions or the utilisation of solvothermal approach. The mechanism proposed for the formation of these structures ${ }^{23}$ engages the generation of seed

\footnotetext{
${ }^{a}$ School of Chemistry, Trinity College Dublin, Dublin 2, Ireland. E-mail: igounko@tcd.ie; Fax: +353-1-6712826;

Tel: +353-1-608 3543

${ }^{b}$ Centre for Research on Adaptive Nanostructures and Nanodevices (CRANN), Trinity College Dublin, Dublin 2, Ireland

${ }^{c}$ Departments of Chemical Engineering, Materials Science and Engineering, Biomedical Engineering, University of Michigan, Ann Arbor, Michigan, 48109, USA

$\dagger$ Electronic supplementary information (ESI) available: Cell cultures experiments, additional spectra, spectroscopic monitoring of the synthesis, additional TEM images, EDX and TGA data. See DOI: $10.1039 / \mathrm{c} 0 \mathrm{cc} 00930 \mathrm{j}$
}

nanocrystals that subsequently act as sites for preferential growth of the arms. Evidence for this mechanism may be found in the production of heterostructures using prepared pre-formed seed of one material followed by the use of additional materials to grow the arms. ${ }^{28,30}$

Here we report new chiral CdS nanotetrapods, which have been produced by heating of a basic aqueous solution of $\mathrm{CdCl}_{2}$, thioacetamide and racemic $(R a c), D$ - or $L$-enantiomeric forms of penicillamine (Pen) under reflux. + Samples of the products have been diluted and examined using UV-VIS and photoluminescence (PL) spectroscopy to analyse the absorbance and emission characteristics of the samples. The absorbance spectra show a defined band edge at approximately $370 \mathrm{~nm}$ for both enantiomers of the stabiliser used as well as for the racemate sample. The PL spectra of the samples (Fig. 1) have shown a very broad emission band between 400 and $700 \mathrm{~nm}$, which is indicative of defect emission. ${ }^{31,32}$

Quantum yields for the samples have been determined using the rhodamine B standard in ethanol ${ }^{33}$ and gave values of up to $12.8 \%$ for the $D$-Pen samples, up to $24.5 \%$ for the $L$-Pen and up to $17 \%$ for Rac-Pen samples.

$C D$ spectra of the $D$ - and $L$-Pen stabilised samples showed clear $\mathrm{CD}$ responses within the band-edge region of the spectrum from 300-400 nm. According to CD results in Fig. 2, the $D$ and $L$-Pen stabilised samples display mirror image spectra involving the band-edge region, while the Rac-Pen samples as expected show no CD activity at all.

Further work was also conducted on analysis of the luminescence lifetimes. It was found that the bi-exponential fit was the most suitable for analysis of these species as has been suggested for analysis of similar defect emitting $\mathrm{CdS}^{31,32}$ with both short ( $D$-Pen $-48.71 \mathrm{~ns}, L$-Pen $-50.66 \mathrm{~ns}$ and $R a c$-Pen $-52.59 \mathrm{~ns}$ ) lifetimes followed by a longer ( $D$-Pen -197.6 ns, L-Pen -196.9 ns and Rac-Pen -198.7 ns) lifetime values.

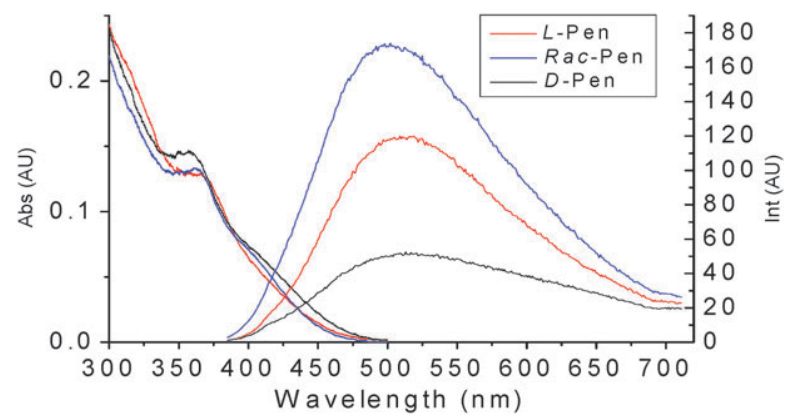

Fig. 1 UV-VIS and PL spectra of Pen stabilised CdS. The spectra were recorded using a SHIMADZU UV-2401PC and a CARY Eclipse spectrometers. 


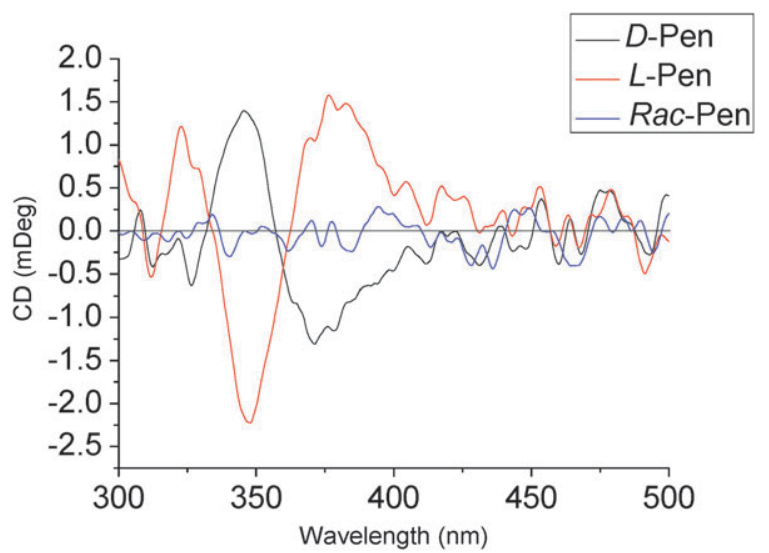

Fig. 2 CD Spectra of penicillamine stabilised CdS. Spectra were recorded using a JASCO J-810 spectropolarimeter.

TEM analysis (Fig. 3) of all samples demonstrated mostly the presence of nanostructures with a tetrapodal morphology, which have tapered arms. The majority of these structures were of $10-20 \mathrm{~nm}$ in diameter. Exactly the same tetrapodal morphology was observed for all $D-, L-$, and $R a c$-Pen samples.

We have also performed energy-dispersive X-ray spectroscopy (EDX) and thermogravimetric analysis (TGA) characterisation of $D$-Pen stabilised CdS samples (see ESI $\dagger$ ). The EDX results have shown an appropriate content of cadmium and sulfur. The occurrence of carbon peaks in the EDX spectra as well as TGA data have also clearly demonstrated the presence of organic (penicillamine) ligands.

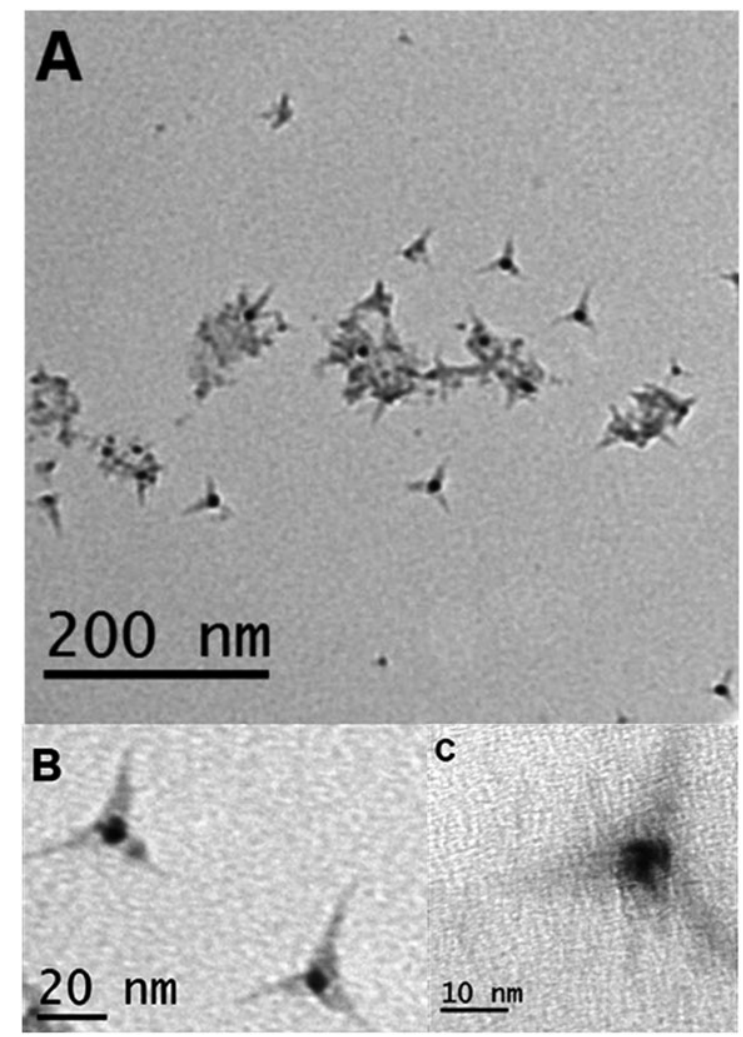

Fig. 3 A wide field TEM images of $D$-Pen nano-tetrapods showing a number of tetrapods (A), a closer look of two tetrapods (B) and a high-resolution image of a single tetrapod (C).

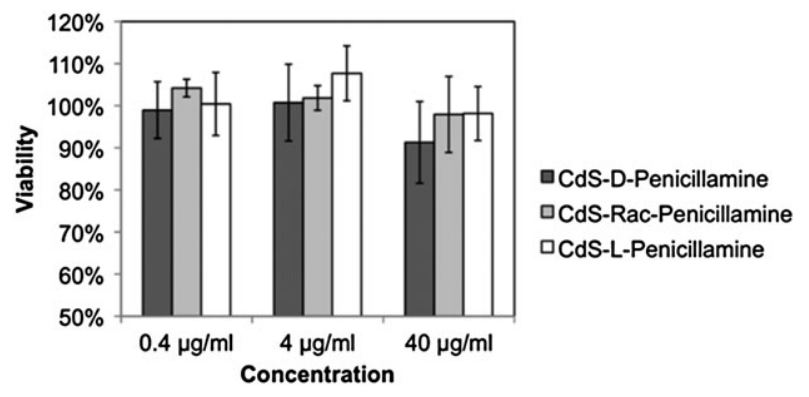

Fig. 4 Histograms showing the evaluation of cell viability depending on tetrapod concentration.

Monitoring of the nano-tetrapod formation by in situ spectroscopic techniques at different times of reflux showed a distinct red-shift in the band-edge which would be symptomatic for the nanoparticle growth and a corresponding decrease in the intensity of the original band (see ESI $\dagger$ ). In the case of the $L$-Pen sample there is a isobastic point which would be indicative of a transformation from the initial small to larger particles. The most interesting result can be seen in the $\mathrm{CD}$ spectra of the tetrapod synthesis monitoring for the $D$ and $L$-Pen stabilised materials. Both of them show a red shift in the main CD peaks as well as a gradual reduction in the strength in the $\mathrm{CD}$ signals. This should be expected if the $\mathrm{CD}$ signals in CdS nano-crystals is originated by surface defects. ${ }^{10}$

We also investigated the cytotoxicity of CdS-penicillamine tetrapods by incubating them at various concentrations $(0.4 \mu \mathrm{g} / \mathrm{ml}, 4 \mu \mathrm{g} / \mathrm{ml}$, and $40 \mu \mathrm{g} / \mathrm{ml})$ for $24 \mathrm{~h}$ with NG108-15 neuroblastoma cell. In general, the tetrapods, regardless of the chirality of penicillamine, produced little to no cytotoxicity in the NG108-15 cells. There was only a slight decrease $(<10 \%)$ in cell viability as the concentration of tetrapods increased 100 folds from $0.4 \mu \mathrm{g} / \mathrm{ml}$ to $40 \mu \mathrm{g} / \mathrm{ml}$ (Fig. 4). Our data indicate that the CdS core of the tetrapods is well protected by penicillamine molecules, producing a nanostructure that is biocompatible and suitable for biological studies.

In conclusion, new chiral light emitting $\mathrm{CdS}$ nano-tetrapods have been prepared using an aqueous synthesis. To the best of our knowledge this is the first reported example of an aqueous heating under reflux leading to the production of semiconducting nano-tetrapods. Preliminary biological testing showed that these materials demonstrate a very low cytotoxicity, that makes them potentially useful for in vitro cellular imaging and biological sensing. Future work will include testing of nanotetrapods as chiral sensors for recognition of various chiral species (drugs, proteins, nucleic acids). We also plan to perform more detailed studies of the photophysical properties of CdS nanotetrapods and the investigation of the electron and energy transfer processes in these systems.

We would like to thank Science Foundation Ireland and CRANN for financial support.

\section{Notes and references}

$\ddagger$ Synthesis: The CdS nano-tetrapods have been prepared as follows. Penicillamine $(0.01 \mathrm{M}, 10 \mathrm{~mL})$ and $\mathrm{CdCl}_{2}(0.01 \mathrm{M}, 8 \mathrm{~mL})$ aqueous solutions were mixed with $40 \mathrm{~mL}$ of millipore water in a $150 \mathrm{~mL}$ round bottomed flask. Then $2 \mathrm{M} \mathrm{NaOH}$ solution was added drop-wise to the mixture in order to adjust the $\mathrm{pH}$ to 11.5-12. After that thioacetamide solution $(0.01 \mathrm{M}, 2 \mathrm{~mL})$ was added to the mixture and it was heated 
under reflux for $2 \mathrm{~h}$. Then the mixture was wrapped in aluminium foil and was allowed to cool to ambient temperature. The solution was concentrated through evaporation of the water under reduced pressure followed by filtration via ultracentrifugation with a $30 \mathrm{kDa}$ MWCO. The precipitate was washed with water-ethanol mixture and then was re-dispersed in millipore water and stored in refrigerator at $+4{ }^{\circ} \mathrm{C}$.

Characterisation: The concentrated purified nanotetrapod samples were diluted by the addition of millipore water and were analysed using UV-VIS on a SHIMADZU UV-2401PC, PL on a CARY Eclipse and CD on a JASCO J-810. TEM images were taken on copper formvar grids using a JEOL, JEM 2100 TEM.

1 P. Alivisatos, Nat. Biotechnol., 2004, 22, 47-52.

2 T. M. Jovin, Nat. Biotechnol., 2003, 21, 32-33.

3 S. J. Byrne, S. A. Corr, T. Y. Rakovich, Y. K. Gun'ko, Y. P. Rakovich, J. F. Donegan, S. Mitchell and Y. Volkov, J. Mater. Chem., 2006, 16, 2896-2902.

4 I. Nabiev, S. Mitchell, A. Davies, Y. Williams, D. Kelleher, R. Moore, Y. K. Gun'ko, S. Byrne, Y. P. Rakovich, J. F. Donegan, A. Sukhanova, J. Conroy, D. Cottell, N. Gaponik, A. Rogach and Y. Volkov, Nano Lett., 2007, 7, 3452-3461.

5 I. L. Medintz, A. R. Clapp, H. Mattoussi, E. R. Goldman, B. Fisher and J. M. Mauro, Nat. Mater., 2003, 2, 630-638.

6 A. L. Rogach, N. Gaponik, J. M. Lupton, C. Bertoni, D. E. Gallardo, S. Dunn, N. L. Pira, M. Paderi, P. Repetto, S. G. Romanov, C. O'Dwyer, C. M. S. Torres and A. Eychmuller, Angew. Chem., Int. Ed., 2008, 47, 6538-6549.

7 H. W. Hillhouse and M. C. Beard, Curr. Opin. Colloid Interface Sci., 2009, 14, 245-259.

8 K. Rajeshwar, N. R. de Tacconi and C. R. Chenthamarakshan, Chem. Mater., 2001, 13, 2765-2782.

9 M. P. Moloney, Y. K. Gun'ko and J. M. Kelly, Chem. Commun., 2007, 3900-3902.

10 S. D. Elliott, M. P. Moloney and Y. K. Gun'ko, Nano Lett., 2008, 8, 2452-2457.

11 T. Nakashima, Y. Kobayashi and T. Kawai, J. Am. Chem. Soc., 2009, 131, 10342-10343.

12 Y. L. Zhou, M. Yang, K. Sun, Z. Y. Tang and N. A. Kotov, J. Am. Chem. Soc., 2010, 132, 6006-6013.
13 A. O. Govorov, Z. Fan, P. Hernandez, J. M. Slocik and R. R. Naik, Nano Lett., 2010, 10, 1374-1382.

14 J. Zhang, M. T. Albelda, Y. Liu and J. W. Canary, Chirality, 2005, 17, 404-418.

15 V. Kitaev, J. Mater. Chem., 2008, 18, 4745-4749.

16 B. Sun, E. Marx and N. C. Greenham, Nano Lett., 2003, 3, 961-963.

17 H. Liu and A. P. Alivisatos, Nano Lett., 2004, 4, 2397-2401.

18 L. Manna, D. J. Milliron, A. Meisel, E. C. Scher and A. P. Alivisatos, Nat. Mater., 2003, 2, 382-385.

19 C. Mauser, T. Limmer, E. Da Como, K. Becker, A. L. Rogach, J. Feldmann and D. V. Talapin, Phys. Rev. B: Condens. Matter Mater. Phys., 2008, 77, 153303.

20 R. G. Xie, U. Kolb and T. Basche, Small, 2006, 2, 1454-1457.

21 J. W. Cho, H. S. Kim, Y. J. Kim, S. Y. Jang, J. Park, J.-G. Kim, Y.-J. Kim and E. H. Cha, Chem. Mater., 2008, 20, $5600-5609$.

22 Y. T. Didenko and K. S. Suslick, J. Am. Chem. Soc., 2005, 127, 12196-12197.

23 S. Kumar and T. Nann, Small, 2006, 2, 316-329.

24 L. Manna, E. C. Scher and A. P. Alivisatos, J. Am. Chem. Soc., 2000, 122, 12700-12706.

25 G. Z. Shen and C. J. Lee, Cryst. Growth Des., 2005, 5, 1085-1089.

26 J. Y. Zhang and W. W. Yu, Appl. Phys. Lett., 2006, 89, 3.

27 S. Sadhu, T. Sen and A. Patra, J. Nanosci. Nanotechnol., 2008, 8, $1238-1243$.

28 D. V. Talapin, J. H. Nelson, E. V. Shevchenko, S. Aloni, B. Sadtler and A. P. Alivisatos, Nano Lett., 2007, 7, 2951-2959.

29 K.-T. Yong, Y. Sahoo, M. T. Swihart and P. N. Prasad, J. Phys. Chem. C, 2007, 111, 2447-2458.

30 A. Fiore, R. Mastria, M. G. Lupo, G. Lanzani, C. Giannini, E. Carlino, G. Morello, M. De Giorgi, Y. Li, R. Cingolani and L. Manna, J. Am. Chem. Soc., 2009, 131, 2274-2282.

31 J. R. Lakowicz, I. Gryczynski, Z. Gryczynski and C. J. Murphy, J. Phys. Chem. B, 1999, 103, 7613-7620.

32 J. R. Lakowicz, I. Gryczynski, G. Piszczek and C. J. Murphy, J. Phys. Chem. B, 2002, 106, 5365-5370.

33 R. F. Kubin and A. N. Fletcher, J. Lumin., 1982, 27, 455-462. 